ARTICLE

Received 13 Nov 2014 | Accepted 24 Nov 2014 | Published 9 Jan $2015 \quad$ DOl: 10.1038/ncomms6950 OPEN

\title{
The evolution of cyclopropenium ions into functional polyelectrolytes
}

Yivan Jiang ${ }^{1, \star}$, Jessica L. Freyer ${ }^{1, \star}$, Pepa Cotanda ${ }^{2,3}$, Spencer D. Brucks ${ }^{1}$, Kato L. Killops ${ }^{4}$, Jeffrey S. Bandar ${ }^{1}$, Christopher Torsitano ${ }^{1}$, Nitash P. Balsara ${ }^{3,5}$, Tristan H. Lambert ${ }^{1} \&$ Luis M. Campos $^{1}$

Versatile polyelectrolytes with tunable physical properties have the potential to be transformative in applications such as energy storage, fuel cells and various electronic devices. Among the types of materials available for these applications, nanostructured cationic block copolyelectrolytes offer mechanical integrity and well-defined conducting paths for ionic transport. To date, most cationic polyelectrolytes bear charge formally localized on heteroatoms and lack broad modularity to tune their physical properties. To overcome these challenges, we describe herein the development of a new class of functional polyelectrolytes based on the aromatic cyclopropenium ion. We demonstrate the facile synthesis of a series of polymers and nanoparticles based on monomeric cyclopropenium building blocks incorporating various functional groups that affect physical properties. The materials exhibit high ionic conductivity and thermal stability due to the nature of the cationic moieties, thus rendering this class of new materials as an attractive alternative to develop ion-conducting membranes.

\footnotetext{
${ }^{1}$ Department of Chemistry, Columbia University, New York, New York 10027, USA. ${ }^{2}$ Joint Center for Artificial Photosynthesis, Lawrence Berkeley National Laboratory, Berkeley, California 94720, USA. ${ }^{3}$ Department of Chemical and Biomolecular Engineering, University of California, Berkeley, California 94720, USA. ${ }^{4}$ Edgewood Chemical Biological Center, Aberdeen Proving Ground, Aberdeen, Maryland 21010, USA. ${ }^{5}$ Materials Sciences Division, Environmental Energy Technologies Division and Joint Center for Energy Storage, Lawrence Berkeley National Laboratory, Berkeley, California 94720, USA. * These authors contributed equally to this work. Correspondence and requests for materials should be addressed to L.M.C. (email: Icampos@columbia.edu).
} 
M odularly designed polymeric materials can be engineered to suit a broad range of applications representing an attractive platform for technological advancement ${ }^{1}$. Materials that possess both inherent compositional versatility and ready accessibility via robust and scalable synthetic pathways are of particular import to the field ${ }^{2,3}$. In this regard, cationic polyelectrolytes have emerged as a versatile class of materials that have been exploited in a broad array of applications ${ }^{4-6}$, ranging from gene delivery ${ }^{7,8}$ to ionconducting membranes ${ }^{9-11}$, and water purification ${ }^{12,13}$. Development in the area of cationic polyelectrolytes has thus far focused on a limited menu of monomeric functionalities, including ammonium, phosphonium, imidazolium, pyridinium and guanidinium ions ${ }^{14-16}$. These heteroatomic systems, while valuable, are application specific and are limited in terms of the ability to finely tune their physical properties. Thus, the identification of new modular cationic polyelectrolytes, with superior characteristics for processing, controllable self-assembly and function, represents an important goal for this field ${ }^{17,18}$.

In developing a new family of polyelectrolytes, certain criteria must be met ${ }^{11,13,17}$ including: (1) thermodynamic stability; (2) ease and scalability of polymerisations by controlled methods; (3) incorporation of accessible chemical handles to allow for diversity and intimate control of physical properties and (4) tunable Coulombic interactions. As an outgrowth of ongoing research efforts, we postulated that polyelectrolytes based on the cyclopropenium ion could satisfy these design criteria, while offering a highly distinct structural architecture and electronic properties. We further recognized that such cyclopropeniumbased systems possess unique characteristics that distinguish them from existing cationic polyelectrolytes, namely: enhanced dispersion of the positive charge (compared with ammonium, phosphonium and guanidinium systems) and weaker H-bond donor capacity (compared with imidazolium and pyridinium ions) $)^{19}$.

As the smallest of the Hückel aromatics ${ }^{20}$, the cyclopropenium (CP) ion possesses significant stability despite its carbocationic nature (Fig. 1a) 11,22 $^{2}$ This remarkable degree of stability may be further enhanced through the incorporation of amino substituents onto the $\mathrm{CP}$ ring ${ }^{23}$. Indeed, with $\mathrm{p} K_{\mathrm{R}}$ values estimated at $>13$, aminocyclopropenium ions are stable even in strongly alkaline aqueous solutions ${ }^{24,25}$. Moreover, thermal decomposition $\left(T_{\mathrm{dec}}\right)$ of the tris(dialkylamino)CP chloride salts has been measured at $>300^{\circ} \mathrm{C}^{19}$, significantly exceeding that of dialkylimidazolium chloride salts $\left(T_{\mathrm{dec}} \sim 250^{\circ} \mathrm{C}\right)^{26}$. These unique structural features have already inspired the development of aminocyclopropenium ions for a range of applications, including as metal ligands ${ }^{27}$, organocatalysts ${ }^{28-30}$ and ionic liquids ${ }^{19}$; however, the incorporation of these cations into a polymeric backbone has only led to polymers with unstable $\mathrm{CP}$ ions as intermediates ${ }^{31}$. Given the tunable functionality and robust, efficient and orthogonal chemistry characterizing CP ions, it is desirable to exploit them in polymeric materials.

Here, we describe the synthesis and evaluation of a new family of cationic polyelectrolytes. As outlined in Fig. 1b, our vision for the design of the parent ionic monomer includes a polymerisable unit, a spectator group (which could also serve as a functional handle) and four additional modular groups that provide the means to tune the physical properties of the resulting macromolecules. In these initial studies, we elected to focus on styrenic $\mathrm{CP}$ monomers (termed, CPR) bearing a series of dialkylamino $\left(\mathrm{NR}_{2}\right)$ substituents. Styrene-based monomers can be subjected to various reversible-deactivation radical polymerization strategies $^{32-34}$. We used reversible-addition fragmentation chain transfer (RAFT) polymerization ${ }^{34}$ to assemble homopolymers, statistical copolymers and diblock copolymers of different a $2 \pi \mathrm{e}^{-}$Hückel aromatic

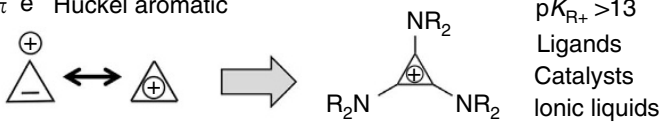

b Polyelectrolytes from cyclopropenium cation buliding blocks.

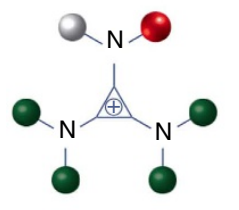

Polymerizable unit

Spectator/functional group

Modular group to tune bulk properties

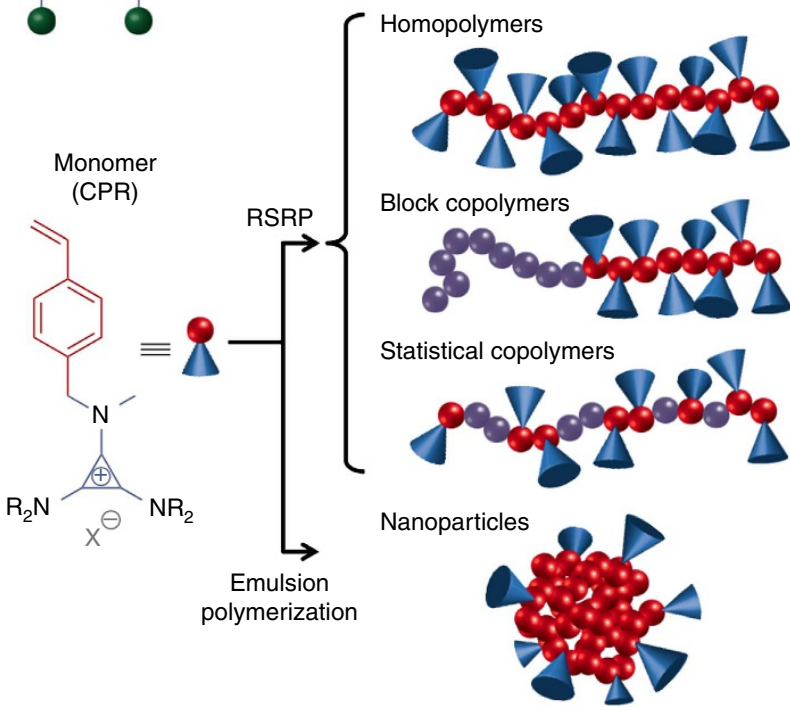

Figure 1 | CP ion building blocks. (a) Structure of the CP ion, including the dialkylamino groups that can be used to stabilize and vary the application of this diverse building block. (b) Types of polyelectrolytes that can be synthesized from CP monomers by reversible-deactivation radical polymerization (RDRP) strategies and emulsion polymerization.

compositions ranging from 20 to $50 \mathrm{~mol} \%$ of CP functionality. As will be shown, we demonstrate that macromolecular assemblies of these materials can be used as ion-conducting membranes, and that the physical properties of these assemblies may be tuned through variation of the dialkylamino handles. In addition, we demonstrate that CPR monomers undergo a surfactant-free emulsion polymerization with styrene, yielding well defined, sub-100 $\mathrm{nm}$ nanoparticles with charged surfaces.

\section{Results}

Monomer synthesis. Our exploration of the CP functional group in the context of cationic polyelectrolytes was originally inspired by its ionic liquid properties ${ }^{19}$ and the straightforward elaboration of the CP ion with various functional groups. Thus far, however, there are no reports on the incorporation of this thermodynamically stable carbocation into macromolecules; CP derivatives have only appeared in polymers as transient species $^{31,35}$. Derivatives of the $\mathrm{CP}$ ion are made from inexpensive reagents and can be easily prepared on a multigram scale under ambient conditions ${ }^{19}$. As robust chemistry is requisite for large-scale production of materials, we devised a viable synthetic strategy en route to the polymerisable $\mathrm{CP}$ ion monomers. The general approach to synthesise CP ioncontaining monomers is based on the facile preparation of asymmetric amino-substituted CP ions (for example, CPR, Fig. 1$)^{36}$. This procedure allows us to intimately change functionality while maintaining cationic properties and thermal stability. Notably, synthetic routes to aminocyclopropenium 
derivatives are modular and highly scalable ${ }^{22}$, with efficiency levels approaching those attained via click chemistry ${ }^{37}$.

The preparation of the CPR monomers begins with pentachlorocyclopropane (1), which is commercially available or inexpensively synthesized in hundred-gram quantities ${ }^{38}$. Reaction of $\mathbf{1}$ with a secondary amine leads to near-quantitative yields of a corresponding CP cation (2 or 3). Thus, amines with high steric hindrance (dicyclohexylamine, $\mathrm{Cy}$ and diisopropylamine, iP) add twice to $\mathbf{1}$, preventing addition of a third bulky amine and leading directly to 2 . Conversely, less sterically hindered amines, such as morpholine (Mo), add thrice to $\mathbf{1}$, resulting in a tris-amino $\mathrm{CP}$ (3). The latter is readily hydrolyzed to its corresponding cyclopropenone in hot, aqueous base, which is subsequently chlorinated to obtain 2 . To underscore the accessibility of these materials, we note that the monomers are obtained by simple purification techniques (see Supplementary Methods for details). Using this process, we prepared multigram quantities of $\mathbf{2}$ incorporating three different secondary amines, as depicted in Fig. 2 (bottom). Importantly, the chemistry in Fig. 2 is highly amenable to a wide range of nucleophilic secondary amines incorporating a variety of functional groups, including elements of asymmetry. We specifically chose to examine dicyclohexylamine, diisopropylamine and morpholine, given that they differ significantly in their degrees of hydrophilicity and steric hindrance.

The synthesis of the CPR monomers from the precursor (2) was readily achieved in $10-20 \mathrm{~g}$ quantities. The chlorinated 1-position of $\mathbf{2}$ is highly susceptible to addition of a secondary amine bearing a polymerisable unit, such as compound $\mathbf{5}$. A styrene-based polymerisable group was chosen as it is a wellbehaved building block in polymer chemistry, and its hydrophobicity relative to the $\mathrm{CP}$ ion moiety could stabilize an emulsion of the type used in nanoparticle synthesis. We postulate that other polymerisable moieties would yield functional monomers as well.

Polymer synthesis and characterization. The three chosen monomers (CPCy, CPiP and CPMo, Fig. 2) were polymerized by RAFT in multigram quantities yielding linear polymers (PCPCy, PCPiP and PCPMo, respectively, Fig. 3). PCPCy was purified through precipitation into 1,4-dioxane from $\mathrm{CH}_{2} \mathrm{Cl}_{2}$ with $88 \%$ recovered yield. PCPiP was precipitated from acetone or $\mathrm{CH}_{2} \mathrm{Cl}_{2}$ into cold ethyl acetate with 70\% recovered yield. Due to their

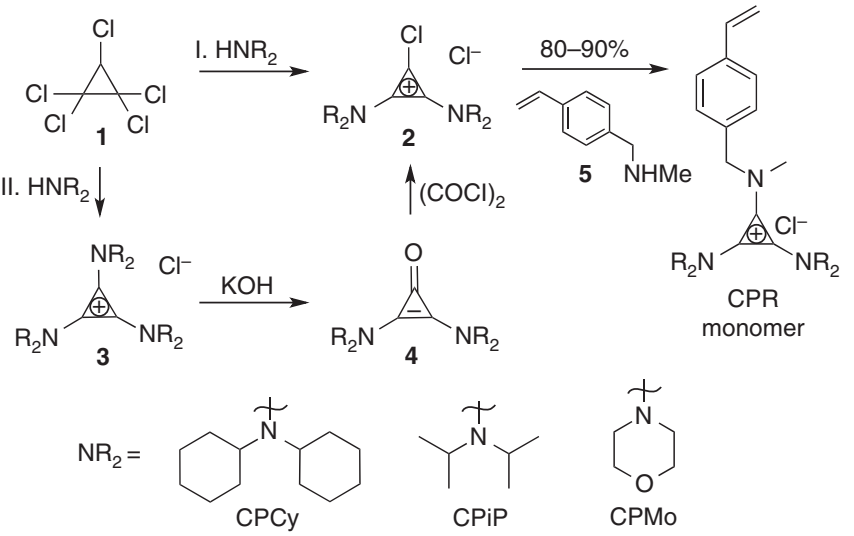

Figure 2 | Synthesis of CP monomers for RAFT polymerisations. The monomers $\mathbf{C P C y}$ and $\mathbf{C P i P}$ were synthesized by addition of dicyclohexylamine (92\%) and diisopropylamine ( $85 \%$ ) to $\mathbf{1}$, followed by substitution of styrenic-type amine $\mathbf{5}$ under basic conditions ( $86 \%$ and $88 \%$, respectively). CPMo was similarly synthesized. After addition of morpholine to $\mathbf{1}$, subsequent hydrolysis and treatment with oxalyl chloride (42\%, 3 steps), 5 was substituted to yield CPMo (59\%). solubility in water, both PCPiP and PCPMo can be purified by dialysis. Purification of PCPMo resulted in a 51\% recovered yield. Each of these polymers was isolated as a powder, and PCPiP and PCPMo were observed to be extremely hygroscopic. Through end-group analysis of the ${ }^{1} \mathrm{H}$ nuclear magnetic resonance (NMR) spectra, we calculated the degree of polymerization (DP) and molecular mass of each of the homopolymers (Supplementary Table 1). Due to the cationic nature of the $\mathrm{CP}$ groups, polymers (and copolymers) cannot be characterized using size exclusion chromatography eluted with organic solvents, as the polymers adhere to the column. We attempted to characterise the dispersity $(\nexists)$ of the hydrophilic homopolymers (PCPiP and PCPMo) on an acetate-buffered aqueous size exclusion chromatography; however, only PCPMo successfully eluted owing to its greater hydrophilicity (Supplementary Fig. 1, Supplementary Table 2). The $\oslash$ of PCPMo was determined to be 1.3, but we note that this value may not accurately reflect the DP control, given that the polyelectrolyte may still be interacting with the column as it is eluted. We note that the synthetic accessibility of these various CP-based polymers is straightforward and highly efficient, rivalling that of ammonium, phosphonium and imidazolium polymers $^{39-42}$.
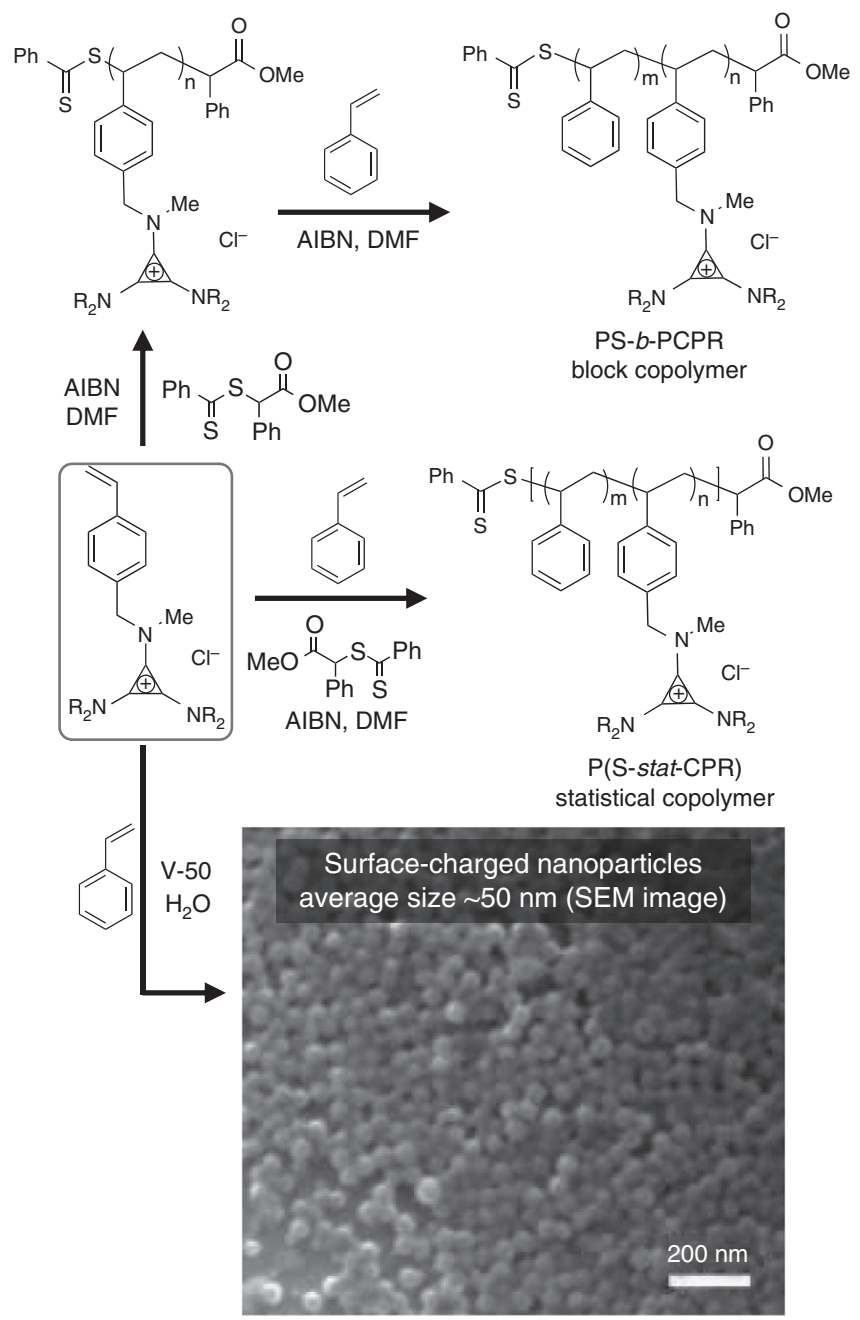

Figure 3 | Synthesis of CP-containing polymers. CPR is polymerized by RAFT yielding both homopolymers, PCPR, and statistical copolymers, P(Sstat-CPR). PCPR is reacted further to form block copolymers PS-b-PCPR of varying styrene content. Nanoparticles are synthesized by surfactant-free emulsion polymerization with styrene using the water-soluble thermal initiator $\mathrm{V}-50$. 
As expected, a significant relationship was observed between the nature of the amino substituent and the physical properties of the resultant homopolymer. We observed that the decomposition temperature $\left(T_{\mathrm{dec}}\right)$, glass transition temperature $\left(T_{\mathrm{g}}\right)$ and solubility of the homopolymers varied as a function of substituent (Supplementary Table 1). Through characterization by thermogravimetric analysis we found that the $T_{\mathrm{dec}}$ of the homopolymers increased as the amino substituents became less sterically hindered. Of particular note, PCPMo decomposed at $310^{\circ} \mathrm{C}$, which is comparable to the $T_{\mathrm{dec}}$ of imidazolium-based polymers ${ }^{43}$. Differential scanning calorimetry was performed to identify the $T_{\mathrm{g}}$ for the homopolymers, as ion-conducting membranes are frequently melt-processed. Both PCPCy and PCPiP have no observable $T_{\mathrm{g}}$ before decomposition, but PCPMo exhibited a $T_{\mathrm{g}}$ of $160^{\circ} \mathrm{C}$. Previous reports have revealed a connection between the nature of the counterion and the accessible temperature window; replacement of the chloride with an alternative, typically bulkier counterion is expected to increase the $T_{\mathrm{dec}}$ while decreasing the $T_{\mathrm{g}}{ }^{19,43,44}$. Importantly, however, our preliminary data clearly demonstrate a similar relationship between alkyl chain identity and observed $T_{\mathrm{dec}}$ and $T_{\mathrm{g}}$; thus, by modifying the CP substituents, we are able to significantly broaden the temperature window in which these materials are processable, without the need to adjust the counterion. In addition, solubility of PCPR homopolymers is highly dependent on amino substituents, again reflecting the influence of building block composition on macromolecular properties. Characterization of the homopolymers, including thermal data and solubility information, is summarized in Supplementary Table 1.

Statistical copolymers were readily synthesized by RAFT, using styrene and CPR monomers, P(S-stat-CPR). When styrene was copolymerized with each monomer in a 1:1 mole ratio, we observed some disparities in the percent incorporation of functional CP monomers in the resulting copolymer. For CPCy, CPiP and CPMo, the degree of incorporation was $~ 50 \%, 48 \%$, and $45 \%$, respectively. Further detailed studies with other monomers are underway to uncover insights into reactivity ratios as a function of R-substituents.

Considering the ability to copolymerize styrene and CPR monomers, we sought to synthesise cationic nanoparticles based on the CPiP monomer via surfactant-free emulsion polymerization. Many traditional strategies rely on the use of surfactants or additional solvents ${ }^{45}$ to obtain sub- $100 \mathrm{~nm}$ cationic particles. By simply mixing styrene and $\mathbf{C P i P}$ at various weight percent values $(1,2.5,5,10$ and $20 \%$ of $\mathbf{C P i P})$ and using a thermally activated radical initiator $(\mathrm{V}-50)$, we were able to obtain particles ranging from 30 to $90 \mathrm{~nm}$ (as characterized by dynamic light scattering Supplementary Fig. 2). Higher loadings of $\mathbf{C P i P}$ compared with styrene resulted in smaller, albeit more disperse, particles. Figure 3 shows the scanning electron microscope image of nanoparticles made from $5 \% \mathbf{C P i P} / 95 \%$ styrene. The average diameter obtained by dynamic light scattering was found to be $50 \mathrm{~nm}$. Furthermore, the particles from stable dipersions, since the zeta potential of the $5 \% \mathbf{C P i P}$ nanoparticles was found to remain above $30 \mathrm{mV}$ over the range of $>10 \mathrm{pH}$ units (Supplementary Fig. 2). As a control, particles synthesized with styrene only (without any surfactants or CPR monomers) were much larger and exhibited a bimodal size distribution (Supplementary Fig. 2). These data demonstrate that the CPiP monomer effectively stabilises oil-in-water droplets, and that the charge is present on the particle surface. A more detailed study of this behaviour will follow, including the incorporation of other CPR monomers into cationic nanoparticles. In general, the ability to make charged nanoparticles in a surfactant-free, large-scale process could have far-reaching potential towards interfacial additives and biological applications ${ }^{46,47}$.
While the CPR monomers were successfully copolymerized to make statistical polymers and nanoparticles, we sought to augment our library of CPR-based macromolecules with block copolyelectrolytes (BCPEs). We synthesized block copolymers PS- $b$-PCPR(CP mol\%) by growing styrene onto PCPR macrochain transfer agents (macro-CTA). By varying the DP of the polystyrene block, we effectively controlled the different mole fractions of the CP functional block. We note that block copolymers PS- $\boldsymbol{b}$-PCPR could also be obtained by the reverse process of growing the functional monomer CPR onto polystyrene macro-CTAs.

Morphology of BCPEs. After synthesizing block copolymers of various compositions, we characterized the morphology of bulk films comprising various CPR-building blocks. Recent studies suggest that nanostructured BCPEs have broad implications in materials chemistry, specifically for fuel cells and batteries, if they undergo microphase segregation. For example, Winey and Elabd recently reported that BCPEs with a lamellar morphology conduct ions more effectively than cationic homopolymers, as water and ions confined within nanochannels may accelerate transport ${ }^{48}$. Computational studies from Olvera de la Cruz and coworkers suggest this effect may be enhanced if the conducting path is a continuous, percolating structure ${ }^{17}$; a microphase segregated morphology of charged and neutral blocks observed in ion-containing block copolymers ${ }^{49}$. With this in mind, we characterized block copolymer samples by small-angle X-ray scattering (SAXS) and TEM to understand microphase segregation in PCPR-containing BCPEs.

In Fig. 4, we show $\mathrm{SAXS}^{50}$ profiles of three representative diblock copolymers. The primary scattering peaks seen in each sample (indicated by filled triangles) is attributed to microphase separation. The scattering profile of PS- $b$-PCPMo(35) contains a higher-order peak at $\boldsymbol{q}=3 \boldsymbol{q}^{*}$. This suggests the presence of a

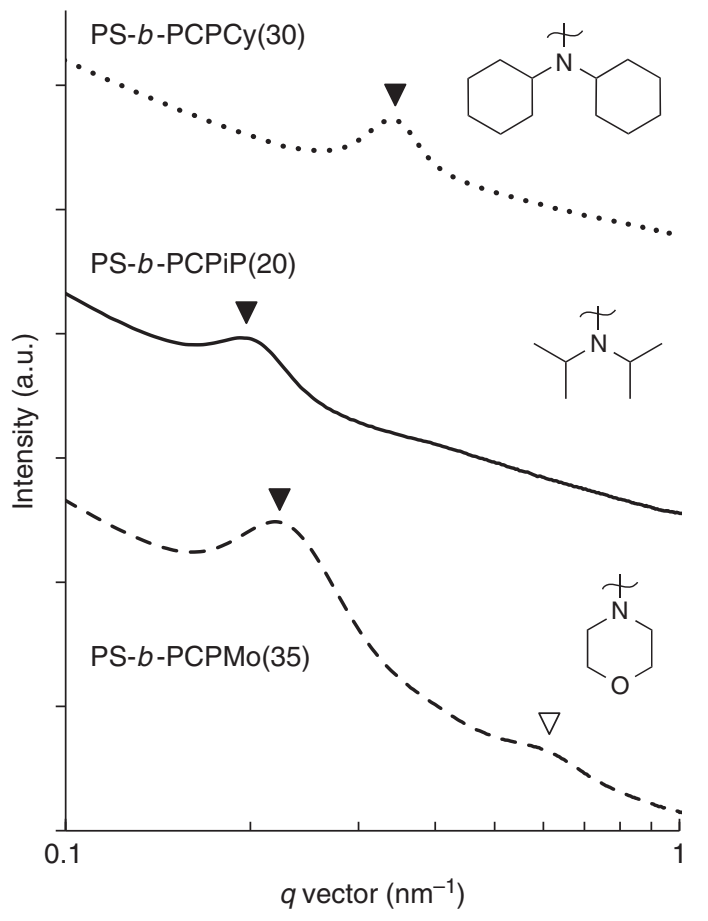

Figure 4 | SAXS profiles of microphase separated diblock copolymers collected at $25^{\circ} \mathbf{C}$. Scattering intensity is plotted as a function of the magnitude of the scattering vector, $\mathbf{q}$. Filled triangles represent the primary scattering peaks and the open triangles represent the higher-order scattering peaks. 
symmetric lamellar phase. The scattering profiles of the other polymers contain only one peak, which indicates a lack of longrange order. The domain spacing, $d$, of the microphase separated diblock copolymers is calculated by the equation $d=2 \pi / \boldsymbol{q}^{\star}$. The domain spacing values corresponding to each diblock copolymer are given in Table 1 . As expected, $d$-spacing increases with molecular mass and molar fraction of styrene. We next sought to probe the potential application of CP-based polyelectrolytes in electrochemical devices 11,17 .

Ion conductivity experiments were performed on PS- $\boldsymbol{b}$ PCPiP(20) using electrochemical impedance spectroscopy. As conductivity is closely related to morphology, we complemented our SAXS experiments with transmission electron microscopy (TEM). A PS-b-PCPiP(20) sample (drop cast, no annealing) was microtomed and imaged by TEM (Fig. 5a,b). Even without

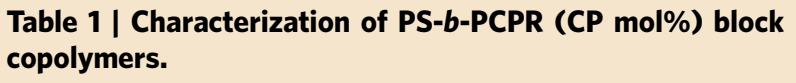

\begin{tabular}{lcccc} 
Sample Name & $\mathbf{M M} \mathbf{k g ~ m o l}^{-\mathbf{1}}$ & $\begin{array}{c}\text { CP\% } \\
\text { by DP }\end{array}$ & $\begin{array}{c}\text { CP\% by } \\
\text { MM }\end{array}$ & $\begin{array}{c}\text { SAXS domain } \\
\text { spacing (nm) }\end{array}$ \\
\hline PS-b-PCPCy(45) & 40 & $45 \%$ & $80 \%$ & 15 \\
PS-b-PCPCy(30) & 50 & $30 \%$ & $70 \%$ & 18 \\
PS-b-PCPiP(50) & 20 & $50 \%$ & $80 \%$ & 18 \\
PS-b-PCPiP(30) & 27 & $30 \%$ & $60 \%$ & 24 \\
PS-b-PCPiP(20) & 30 & $20 \%$ & $50 \%$ & 31 \\
PS-b-PCPMo(50) & 38 & $50 \%$ & $80 \%$ & 26 \\
PS-b-PCPMo(35) & 42 & $35 \%$ & $70 \%$ & 28
\end{tabular}

$\mathrm{CP}$, cyclopropenium; DP, degree of polymerization; NMR, nuclear magnetic resonance; $\mathrm{SAXS}$, small-angle X-ray scattering

Block copolymers were synthesized by the addition of styrene to the three homopolymers, and domain spacing was calculated by SAXS (Supplementary Fig. 3). Molecular mass (MM) was determined by ${ }^{1} \mathrm{H}$ NMR spectroscopy. staining, we clearly observe microphase segregation (cylindrical morphology) (Supplementary Fig. 4). Staining with $\mathrm{RuO}_{4}$ vapour for $2 \mathrm{~min}$ preferentially stains the cationic block and helps to visualize the internal structure. The electron micrographs obtained (Fig. 5a,b) show hexagonally packed cylinders in different orientations. The domain spacing by TEM was $29 \mathrm{~nm}$, which is consistent with the domain spacing determined by SAXS $(31 \mathrm{~nm}$; see Table 1$)$. The lighter colour of the cylinders with respect to the matrix in Fig. 5a,b indicates that PS cylinders are embedded in a PCPiP matrix. The stained PCPiP block scatters more electrons, and therefore appears darker by TEM (see Supplementary Fig. 4). The non-functionalised PS cylinders in Fig. 5a,b occupy a very large fraction of the image because the mole fraction of the functional block in this polymer is only $20 \%$. The continuous nature of the conducting phase matrix observed in charged diblock copolymers is expected to facilitate ion transport ${ }^{49}$, and is consistent with the ideal percolating structure found by computational modelling ${ }^{17}$.

\section{Discussion}

The in-plane conductivity, $\sigma$, of $\mathbf{P S}-\boldsymbol{b}$-PCPiP(20) equilibrated in humid air with $90 \%$ relative humidity $(\mathrm{RH})$ was measured as a function of increasing temperature from 25 to $65^{\circ} \mathrm{C}$ (Fig. 5c). To ensure equilibration, samples were initially annealed for 1 week at $90 \% \mathrm{RH}$ at $25^{\circ} \mathrm{C}$ and for $48 \mathrm{~h}$ at each subsequent temperature of interest. The straight line in Fig. $5 \mathrm{c}$ is the least-squares fit through the equilibrated conductivity data at each temperature value. In principle, the change in conductivity with temperature, shown in Fig. $5 c$, could either be due to changes in the mobility of chloride ions or to a change in ion concentration in the membrane. The fact that ion concentration in the membranes is constant indicates that the slope of the line provides an estimate

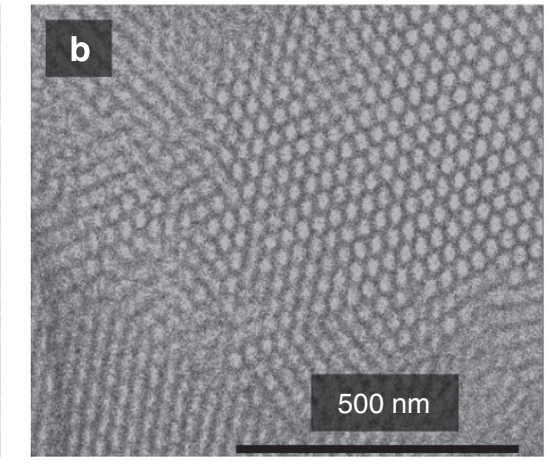

C
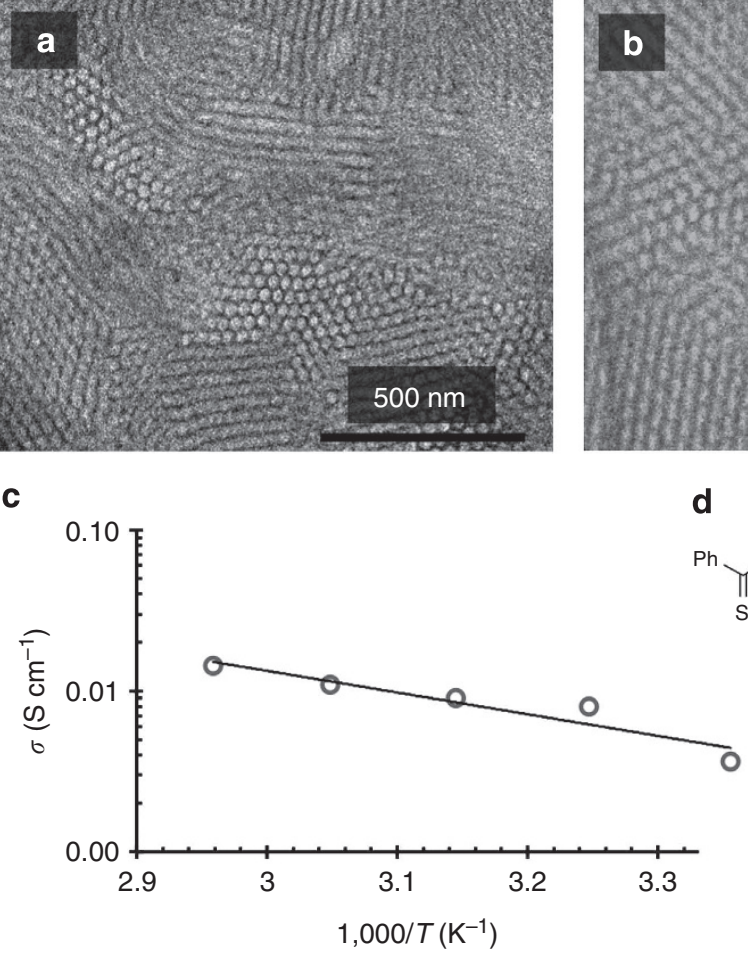

d<smiles>COC(=O)C(CCC(C)c1ccc(CN(C)C2=C(N(C(C)C)C(C)C)N2C(C)C)cc1)C(C)c1ccccc1</smiles>

Figure 5 | Morphology and ionic conductivity of bulk PS-b-PCPiP films. (a and $\mathbf{b}$ ) Two representative TEM images of PS-b-PCPiP(20) reveal a morphology of hexagonally packed cylinders ( $d$-spacing $=29 \mathrm{~nm}$; the light colour corresponds to PS). (c) lonic conductivity as a function of inverse temperature, from 25 to $65^{\circ} \mathbf{C}$, for (d) PS-b-PCPiP(20) (ion-exchange capacity, IEC $=1.3$, at $90 \% \mathrm{RH}$ ). 
of the activation energy for transport of chloride ions through the membrane (Arrhenius law). The estimated activation energy for this system is $25 \mathrm{~kJ} \mathrm{~mol}^{-1}$. This value is comparable to that reported previously for the imidazolium-containing diblock copolymer analogue in water, poly(styrene- $b$-4-vinylbenzyltrimethylimidazolium chloride) (PS- $b$-PIm(35)), $27 \mathrm{~kJ} \mathrm{~mol}^{-1}$ (ref. 43). However, at room temperature, the conductivity of the PS- $b$-PCPiP(20) polymer (ion-exchange capacity, IEC $=$ $1.3 \mathrm{meq} \mathrm{g}^{-1}$ ) is rather high, $0.004 \mathrm{~S} \mathrm{~cm}^{-1}$, considering the low water uptake, $\lambda_{\mathrm{w}}=7$, of this membrane $\left(\lambda_{\mathrm{w}}\right.$ is the number of water molecules per chloride ion in the membrane). This value of $\lambda_{\mathrm{w}}$ is four times lower than the value obtained for PS- $b-\operatorname{PIm}(35)$ immersed in water, for the same conductivity $\left(\lambda_{\mathrm{w}}=30\right.$, $\left.\sigma=0.004 \mathrm{~S} \mathrm{~cm}^{-1}\right)$, and higher IEC $\left(2.1 \mathrm{meqg}^{-1}\right)^{43}$. These results indicate that the $\mathrm{CP}$-based polyelectrolytes conduct ions more effectively than the optimized membranes from imidazolium-containing polymers, with a minimum amount of water present. Further tuning of the functional groups, backbone structure and morphology is expected to result in polyelectrolytes with exceptionally high ion conductivities ${ }^{51-54}$.

In conclusion, we have introduced a new family of electronrich cationic polyelectrolytes based on the $\mathrm{CP}$ ion building block. The robust, efficient and orthogonal chemistry to synthesise the monomers provides facile access to a variety of polymers by RAFT and to well-defined cationic nanoparticles by surfactant-free emulsion polymerization. The nanoparticles exhibit high charge density on the surface and stability over a wide range of $\mathrm{pH}$ values. The family of linear polymers is characterized by widely variable physical properties, which are highly dependent on the amino substituents flanking the aromatic cation. Through TEM and SAXS measurements, we observed microphase segregation in bulk samples of the BCPE. In diblock copolymers, the domain spacing increased with increasing styrene content (the length of the functional block was fixed). Compared with imidazolium analogues, CP-based BCPEss show superior properties as ion conductive materials, and we anticipate that further optimization will lead to improved performance. Future studies will be aimed at probing the structure-property relationships of these materials by expanding our PCPR library through adjustment of the polymer backbone, modular functional groups, block copolymer composition and CP counterion. Moreover, other CPR monomers will be incorporated into nanoparticles via the one-pot emulsion polymerization to assess their efficacy in various biomedical applications and membrane technologies. With such modularity, this new class of CP-based polyelectrolytes offers a wealth of functionality that translates to significant potential across a broad array of applications.

\section{Methods \\ Synthesis of $\mathbf{N}$-methyl-1-(2,3-bis(dicyclohexylamino)cyclopropenium)-4- vinylbenzylamine chloride (CPCy). To a dry round bottom flask of 2,3-bis(di- cyclohexylamino)-1-chlorocyclopropenium chloride $(22.1 \mathrm{~g}, 47.3 \mathrm{mmol}, 1.0$ equiv) under argon was added $\mathrm{CH}_{2} \mathrm{Cl}_{2}(150 \mathrm{ml})$ and triethylamine $(6.54 \mathrm{ml}, 47.3 \mathrm{mmol}$, 1.0 equiv). $\mathrm{N}$-Methyl-4-vinylbenzylamine (7.74 g, $47.3 \mathrm{mmol}$ and 1.0 equiv) was then slowly added to the solution and the reaction was stirred overnight. $\mathrm{CH}_{2} \mathrm{Cl}_{2}$ $(700 \mathrm{ml})$ was added and the mixture was washed with $1 \mathrm{M} \mathrm{HCl}(3 \times 200 \mathrm{ml})$ and brine $(1 \times 200 \mathrm{ml})$, dried with anhydrous sodium sulfate and concentrated in vacuo to yield a crude solid. The crude product was purified with silica gel chromato- graphy (EtOAc then $5 \%$ iPrOH in $\mathrm{CH}_{2} \mathrm{Cl}_{2}$ ) to yield an off-white solid $(23.5 \mathrm{~g}$, $40.6 \mathrm{mmol}, 86 \%$ yield). Monomers were characterized by ${ }^{1} \mathrm{H}$ and ${ }^{13} \mathrm{C}$ NMR (see Supplementary Figs 5-10), and all new compounds were characterized by NMR and mass spectrometry (see Supplementary Notes and Methods).}

Sample synthesis of PCPR: synthesis of PCPCy. To a dry $20 \mathrm{ml}$ (see Supplementary Notes and Methods for details) scintillation vial, CPCy (6.0 g, $10.4 \mathrm{mmol}, 60.0$ equiv), methyl 2-(phenylcarbonothioylthio)-2-phenylacetate (52.3 mg, $1.73 \mathrm{mmol}, 1.0$ equiv), AIBN ( $4.3 \mathrm{mg}, 0.26 \mathrm{mmol}, 0.15$ equiv) and DMF
$(6.0 \mathrm{ml})$ were added and vortexed to form a homogenous solution. This solution was transferred to a flame-dried ampule. After 4 freeze-pump-thaw cycles, the ampule was sealed under vacuum. The polymerization was run for $12 \mathrm{~h}$ at $80^{\circ} \mathrm{C}$ with vigorous stirring. The reaction mixture was precipitated from $\mathrm{CH}_{2} \mathrm{Cl}_{2}$ into 1,4-dioxane three times to remove monomer. The polymer was then precipitated an additional three times into hexanes to remove residual 1,4-dioxane. Drying in vacuo yielded the pure polymer as a pink powder $(5.3 \mathrm{~g}, 88 \%$ yield). Homopolymers were characterized by ${ }^{1} \mathrm{H}$ NMR (see Supplementary Figs 11-13).

Small-angle X-ray scattering. Thick polymer samples $(1 \mathrm{~mm})$ were prepared by pressing the powder into a teflon washer. Synchrotron SAXS measurements were performed using the 7.3.3 beamline at the Advanced Light Source (ALS, Lawrence Berkeley National Laboratory). The wavelength $\lambda$ of the incident X-ray beam was $0.124 \mathrm{~nm}\left(\Delta \lambda / \lambda=10^{-4}\right)$ and a sample-to-detector distance of $4 \mathrm{~m}$. The resulting twodimensional scattering data were averaged azimuthally to obtain intensity versus magnitude of the scattering wave vector $\boldsymbol{q}(\boldsymbol{q}=4 \pi \sin (\theta / 2) / \lambda$, where $\theta$ is the scattering angle). All of the scattering profiles were azimuthally symmetric. The scattering data were corrected for the detector dark current and the scattering from air and Kapton windows.

Electrochemical impedance spectroscopy. In-plane chloride conductivity of hydrated membranes with dimensions $2 \mathrm{~cm} \times 1 \mathrm{~cm} \times 450 \mu \mathrm{m}$ was measured by AC impedance spectroscopy using platinum electrodes in the standard four probe configuration using a BekkTech sample clamp. Polymer films of PS-b-PCPiP(20) were prepared by drop casting a $100 \mathrm{mg} \mathrm{ml}^{-1}$ solution of polymer onto a clean Teflon substrate. In-plane chloride conductivity of a hydrated membrane composed of PS- $\boldsymbol{b}$-PCPiP(20) (calculated molecular mass $=31 \mathrm{KDa}, \mathrm{DP}=174$ ) with dimensions $2 \mathrm{~cm} \times 1 \mathrm{~cm} \times 450 \mu \mathrm{m}$ was measured by AC impedance spectroscopy using platinum electrodes in the standard four probe configuration using a BekkTech sample clamp. Conductivities were collected under humidified conditions, and temperature and $\mathrm{RH}$ were controlled by an environmental chamber (Qualitest). Data were collected using $10 \mathrm{mV}$ amplitude over a frequency range of $1 \mathrm{~Hz}-10 \mathrm{MHz}$. Separate experiments were conducted to ensure that the response of the sample was linear in this window. Samples were annealed at the temperature of interest for $24-48 \mathrm{~h}$ until the measured impedance did not change. Conductivity, $\sigma$, is given by equation (1):

$$
\sigma=\frac{w}{r S}
$$

where $S$ is the cross-sectional area of sample film, $r$ is the intercept of the Nyquist semi-circle on the real axis $(\Omega)$ and $w$ is the distance between the inner platinum electrodes.

Transmission electron microscopy. Films of PS- $\boldsymbol{b}$-PCPiP(20) (calculated molecular mass $=33.4 \mathrm{KDa}, \mathrm{DP}=200$ ) were prepared by drop casting a $100 \mathrm{mg} \mathrm{ml}^{-1}$ solution of polymer onto a clean Teflon substrate. After allowing to dry for $24 \mathrm{~h}$, the film was sectioned with Leica UltraCut 6 ultramicrotome at $-40^{\circ} \mathrm{C}$, nominal thickness $70 \mathrm{~nm}$ using a Diatome Cryo $35^{\circ}$ diamond knife. Sections were placed on 300 mesh copper grids with homemade lacey carbon film on top. The sections were stained with $\mathrm{RuO} 4$ vapour for $2 \mathrm{~min}$, which preferentially stained the PCPiP block. Sections were imaged with FEI Tecnai F20 TEM operated at $200 \mathrm{kV}$. Images were analysed using ImageJ $1.48 \mathrm{v}$ software.

\section{References}

1. Hawker, C. J. \& Wooley, K. L. The convergence of synthetic organic and polymer chemistries. Science 309, 1200-1205 (2005).

2. Hunt, J. N. et al. Tunable, High modulus hydrogels driven by ionic coacervation. Adv. Mater. 23, 2327-2331 (2011).

3. Leibfarth, F. A. et al. A facile route to ketene-functionalized polymers for general materials applications. Nat. Chem. 2, 207-212 (2010)

4. Lodge, T. P. A unique platform for materials design. Science 321, 50-51 (2008).

5. Gao, R., Wang, D., Heflin, J. R. \& Long, T. E. Imidazolium sulfonate-containing pentablock copolymer-ionic liquid membranes for electroactive actuators. J. Mater. Chem. 22, 13473-13476 (2012).

6. Hallinan, D. T. \& Balsara, N. P. Polymer electrolytes. Annu. Rev. Mater. Res. 43, 503-525 (2013).

7. De Smedt, S., Demeester, J. \& Hennink, W. Cationic polymer based gene delivery systems. Pharm. Res. 17, 113-126 (2000).

8. Samal, S. K. et al. Cationic polymers and their therapeutic potential. Chem. Soc. Rev. 41, 7147-7194 (2012).

9. Pan, J., Chen, C., Zhuang, L. \& Lu, J. Designing advanced alkaline polymer electrolytes for fuel cell applications. Acc. Chem. Res. 45, 473-481 (2011).

10. Hickner, M. A., Herring, A. M. \& Coughlin, E. B. Anion exchange membranes: current status and moving forward. J. Polym. Sci. B Polym. Phys. 51, 1727-1735 (2013).

11. Chen, Y. et al. Enhancement of anhydrous proton transport by supramolecular nanochannels in comb polymers. Nat. Chem. 2, 503-508 (2010). 
12. Elimelech, M. \& Phillip, W. A. The future of seawater desalination: energy, technology, and the environment. Science 333, 712-717 (2011).

13. Gin, D. L. \& Noble, R. D. Designing the next generation of chemical separation membranes. Science 332, 674-676 (2011).

14. Jangu, C. \& Long, T. E. Phosphonium cation-containing polymers: from ionic liquids to polyelectrolytes. Polymer 55, 3298-3304 (2014).

15. Qiu, B., Lin, B., Qiu, L. \& Yan, F. Alkaline imidazolium- and quaternary ammonium-functionalized anion exchange membranes for alkaline fuel cell applications. J. Mater. Chem. 22, 1040-1045 (2012).

16. Yuan, J., Mecerreyes, D. \& Antonietti, M. Poly(ionic liquid)s: an update. Prog. Polym. Sci. 38, 1009-1036 (2013).

17. Sing, C. E., Zwanikken, J. W. \& Olvera de la Cruz, M. Electrostatic control of block copolymer morphology. Nat. Mater. 13, 694-698 (2014).

18. Steele, B. C. H. \& Heinzel, A. Materials for fuel-cell technologies. Nature 414, 345-352 (2001)

19. Curnow, O. J., MacFarlane, D. R. \& Walst, K. J. Triaminocyclopropenium salts as ionic liquids. Chem. Commun. 47, 10248-10250 (2011).

20. Hückel, E. Grundzüge der Theorie ungesättiger und aromatischer Verbindungen. Z. Physik 70, 77-85 (1938).

21. Breslow, R. Synthesis of the s-triphenylcyclopropenyl cation. J. Am. Chem. Soc. 79, 5318-5318 (1957).

22. Bandar, J. S. \& Lambert, T. H. Aminocyclopropenium ions: synthesis, properties, and applications. Synthesis 45, 2485-2498 (2013).

23. Yoshida, Z. \& Tawara, Y. Aminocyclopropenium ion. J. Am. Chem. Soc. 93, 2573-2574 (1971)

24. Yoshida, Z.-I., Tawara, Y., Hirota, S. \& Ogoshi, H. Vibrational spectrum of trisdimethylaminocyclopropenium perchlorate. Bull. Chem. Soc. Jpn 47, 797800 (1974).

25. Kerber, R. C. \& Hsu, C.-M. Substituent effects on cyclopropenium ions. J. Am. Chem. Soc. 95, 3239-3245 (1973).

26. Huddleston, J. G. et al. Characterization and comparison of hydrophilic and hydrophobic room temperature ionic liquids incorporating the imidazolium cation. Green Chem. 3, 156-164 (2001).

27. Bruns, H. et al. Synthesis and coordination properties of nitrogen(i)-based ligands. Angew. Chem. Int. Ed. 49, 3680-3683 (2010).

28. Bandar, J. S. \& Lambert, T. H. Enantioselective brønsted base catalysis with chiral cyclopropenimines. J. Am. Chem. Soc. 134, 5552-5555 (2012).

29. Bandar, J. S. \& Lambert, T. H. Cyclopropenimine-catalyzed enantioselective mannich reactions of tert-butyl glycinates with n-boc-imines. J. Am. Chem. Soc. 135, 11799-11802 (2013).

30. Wilde, M. M. D. \& Gravel, M. Bis(amino)cyclopropenylidenes as organocatalysts for acyl anion and extended umpolung reactions. Angew. Chem. Int. Ed. 52, 12651-12654 (2013).

31. Weidner, C. H. \& Long, T. E. Synthesis and characterization of 3-aryl-2(polystyryl)cyclopropenones via cyclopropenium ion substitution on polystyrene. J. Polym. Chem. A Polym. Chem. 33, 1-6 (1995).

32. Hawker, C. J., Bosman, A. W. \& Harth, E. New polymer synthesis by nitroxide mediated living radical polymerizations. Chem. Rev. 101, 3661-3688 (2001).

33. Matyjaszewski, K. \& Xia, J. Atom transfer radical polymerization. Chem. Rev. 101, 2921-2990 (2001).

34. Chiefari, J. et al. Living free-radical polymerization by reversible additionfragmentation chain transfer: the RAFT process. Macromolecules 31, 55595562 (1998).

35. Peart, P. A. \& Tovar, J. D. Poly(cyclopropenone)s: formal inclusion of the smallest Hückel aromatic into $\pi$-conjugated polymers. J. Org. Chem. 75, 5689-5696 (2010).

36. Curnow, O. J., Holmes, M. T., Ratten, L. C., Walst, K. J. \& Yunis, R. A facile route to functionalised, protic and chiral ionic liquids based on the triaminocyclopropenium cation. RSC Adv. 2, 10794-10797 (2012).

37. Kolb, H. C., Finn, M. G. \& Sharpless, K. B. Click chemistry: diverse chemical function from a few good reactions. Angew. Chem., Int. Ed. 40, 2004-2021 (2001).

38. Tobey, S. W. \& West, R. Pentachlorocyclopropane. J. Am. Chem. Soc. 88, 2478-2481 (1966)

39. Hemp, S. T. et al. Comparing ammonium and phosphonium polymerized ionic liquids: thermal analysis, conductivity, and morphology. Macromol. Chem. Phys. 214, 2099-2107 (2013).

40. Yuan, J. \& Antonietti, M. Poly(ionic liquid)s: polymers expanding classical property profiles. Polymer 52, 1469-1482 (2011).

41. Wang, R. \& Lowe, A. B. RAFT polymerization of styrenic-based phosphonium monomers and a new family of well-defined statistical and block polyampholytes. J. Polym. Sci. A Polym. Chem. 45, 2468-2483 (2007).

42. Texter, J. Anion responsive imidazolium-based polymers. Macromol. Rapid Commun. 33, 1996-2014 (2012).

43. Sudre, G., Inceoglu, S., Cotanda, P. \& Balsara, N. P. Influence of bound ion on the morphology and conductivity of anion-conducting block copolymers. Macromolecules 46, 1519-1527 (2013).
44. Weber, R. L. et al. Thermal and ion transport properties of hydrophilic and hydrophobic polymerized styrenic imidazolium ionic liquids. J. Polym. Sci. B Polym. Phys 49, 1287-1296 (2011).

45. Ramos, J., Forcada, J. \& Hidalgo-Alvarez, R. Cationic polymer nanoparticles and nanogels: from synthesis to biotechnological applications. Chem. Rev. 114, 367-428 (2013).

46. Rothberg, J. M. et al. An integrated semiconductor device enabling non-optical genome sequencing. Nature 475, 348-352 (2011).

47. Nederberg, F. et al. Biodegradable nanostructures with selective lysis of microbial membranes. Nat. Chem. 3, 409-414 (2011).

48. Ye, Y., Sharick, S., Davis, E. M., Winey, K. I. \& Elabd, Y. A. High hydroxide conductivity in polymerized ionic liquid block copolymers. ACS Macro Lett. 2 , 575-580 (2013).

49. Park, M. J. et al. Increased water retention in polymer electrolyte membranes at elevated temperatures assisted by capillary condensation. Nano Lett. 7, 3547-3552 (2007).

50. Alexander, H. et al. A SAXS/WAXS/GISAXS beamline with multilayer monochromator. J. Phys. Conf. Ser 247, 012007 (2010).

51. Schmidt-Rohr, K. \& Chen, Q. Parallel cylindrical water nanochannels in Nafion fuel-cell membranes. Nat. Mater. 7, 75-83 (2008).

52. Choi, J.-H., Ye, Y., Elabd, Y. A. \& Winey, K. I. Network structure and strong microphase separation for high ion conductivity in polymerized ionic liquid block copolymers. Macromolecules 46, 5290-5300 (2013).

53. Hoarfrost, M. L., Tyagi, M. S., Segalman, R. A. \& Reimer, J. A. Effect of confinement on proton transport mechanisms in block copolymer/ionic liquid membranes. Macromolecules 45, 3112-3120 (2012).

54. Ye, Y., Choi, J.-H., Winey, K. I. \& Elabd, Y. A. Polymerized ionic liquid block and random copolymers: effect of weak microphase separation on ion transport. Macromolecules 45, 7027-7035 (2012).

\section{Acknowledgements}

This work was supported in part by the National Science Foundation (CAREER, DMR1351293 to L.M.C. and CHE-0953259 to T.H.L.), ACS Petroleum Research Fund (54471 DNI7 to L.M.C.), and 3M Corporation (to L.M.C.). Y.J. thanks the Columbia Amgen Scholars Program and the Columbia Science Research Fellows. J.S.B is grateful for NDSEG and NSF fellowships. K.L.K. thanks the Army Basic Research Program and ECBC for financial support. Transmission electron micrographs were collected at NYSBC, and were made possible by a grant from NYSTAR. The investigation was conducted in a facility constructed with support from Research Facilities Improvement Program Grant number C06 RR017528-01-CEM from the National Center for Research Resources, National Institutes of Health. This material is in part based upon work performed by the Joint Center for Artificial Photosynthesis, a DOE Energy Innovation Hub, supported through the Office of Science of the U.S. Department of Energy under Award Number DE-SC0004993. SAXS experiments were performed at Lawrence Berkeley National Laboratory's Advance Light Source, Beamline 7.3.3. Beamline 7.3.3 of the Advanced Light Source is supported by the Director of the Office of Science, Office of Basic Energy Sciences, of the U.S. Department of Energy under Contract No. DE-AC02 05CH11231. We thank Dr Jos Paulusse for performing size exclusion chromatography on the homopolymers. We also thank Qi Jiang for performing thermogravimetric analysis on the homopolymers.

\section{Author contributions}

All authors contributed to the conception and execution of experiments. Materials were synthesized by J.L.F., Y.J., S.D.B. and K.L.K. Ionic conductivity measurements and X-ray scattering were performed by P.C. All authors contributed to data analysis. The paper was written by J.L.F., Y.J. and L.C., with input from all authors.

\section{Additional information}

Accession codes: A patent related to this research has been issued, 'Cyclopropenium Polymers and Methods for Making the Same,' WO 2014/022365 A1.

Supplementary Information accompanies this paper at http://www.nature.com/ naturecommunications

Competing financial interests: The authors declare no competing financial interests

Reprints and permission information is available online at http://npg.nature.com/ reprintsandpermissions/

How to cite this article: Jiang, Y. et al. The evolution of cyclopropenium ions into functional polyelectrolytes. Nat. Commun. 6:5950 doi: 10.1038/ncomms6950 (2015).

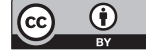

This work is licensed under a Creative Commons Attribution 4.0 International License. The images or other third party material in this article are included in the article's Creative Commons license, unless indicated otherwise in the credit line; if the material is not included under the Creative Commons license, users will need to obtain permission from the license holder to reproduce the material To view a copy of this license, visit http://creativecommons.org/licenses/by/4.0/ 